\title{
FAUNA DE ABELHAS EUGLOSSINI (HYMENOPTERA: APIDAE) EM DOIS FRAGMENTOS DE MATA ATLÂNTICA NO SUL DO ESPÍRITO SANTO
}

\author{
Josélia Luiza Pirovani ${ }^{1}$ \\ Alexandra Aparecida Zorzal ${ }^{2}$ \\ Patrícia Batista de Oliveira ${ }^{3}$ \\ Luceli de Souza ${ }^{4}$
}

\begin{abstract}
Resumo: O objetivo principal desse trabalho foi conhecer a fauna de abelhas Euglossini em dois fragmentos de Mata Atlântica em dois Parques Estaduais localizadas no sul do Espírito Santo. Para a captura dos indivíduos, utilizou-se iscas de chumaços de algodão envolvidos em gaze e presos no galho a 1,5m de altura utilizando quatro iscas aromáticas: acetato de benzila, baunilha, eucaliptol e eugenol. Os indivíduos foram capturados com rede entomológica, mortas com acetato de etila e transferidas para tubos com o nome da essência e transportadas para o Laboratório de Zoologia do CCENS/UFES, preparadas e identificadas com auxilio de literatura. Foram realizadas coletas de setembro de 2014 a agosto de 2015. Foram coletados 232 indivíduos pertencentes a três gêneros. O gênero mais abundante foi Euglossa com 139 exemplares, seguido de Eulaema com 81 indivíduos e Eufriesea com 12 indivíduos. A essência mais atrativa foi eucaliptol (78,87\%), que atraiu 183 machos, corroborando com a literatura, seguida por acetato de benzila (11,20\%, $n=26$ machos), baunilha (7,32\%, $n=17)$, e eugenol $(2,58 \%, n=6)$.
\end{abstract}

Palavras-chave: Orquídeas; Essências; Desmatamento.

\footnotetext{
1 Ciências Biológicas Licenciatura/Universidade Federal do Espírito Santo, Brasil. E-mail: joseliapirovani@gmail.com.

2 Ciências Biológicas Licenciatura/Universidade Federal do Espírito Santo, Brasil. E-mail: alexandra.zorzal@gmail.com.

3 Ciências Biológicas Licenciatura/Universidade Federal do Espírito Santo, Brasil. E-mail: patricia.bio77@gmail.com.

4 Ciências Biológicas Licenciatura/Universidade Federal do Espírito Santo, Brasil. E-mail: lucelidesouza@yahoo.com.br.
} 